hep-ph 9311355

EP-CPTh.A274.1293

LAVAL-PHY-13-93

\title{
New classes of summable Skyrmions
}

\author{
Luc Marleau \\ Centre de Physique Théorique,École Polytechnique \\ 91128 \\ Palaiseau \\ CEDEX, \\ France
}

(October 1993)

\begin{abstract}
We introduce two new classes of summable Skyrmions. The Lagrangians they originate from are explicitely constructed. We also analyse how some models could be solve. Exact solutions are found for Skyrme-like toy models.
\end{abstract}

PACS number(s): 11.30.Na, 11.30.Rd, 12.15.Cc.

Typeset using REVTEX 


\section{INTRODUCTION}

The Lagrangian originally proposed by Skyrme [1] is composed of a kinetic term and a self-interaction term of order two and four, respectively, in the derivatives of the pion field. The second term prevents the model from being renormalizable, but it induces pion field configurations which are soliton solutions (Skyrmions). These are interpreted as baryons and a number of their properties can be computed leading to many successfull predictions. The $1 / N_{c}$ analysis however suggests that if an effective Lagrangian is to represent accurately the low-energy limit of QCD, it should behave as an effective theory of infinitely many mesons and include all orders of derivative of the fields [2]. In that sense, the Skyrme model represent a naive (order-four) attempt to provide such a description. The exact form of such the low-energy effective Lagrangian is for the moment out our reach and indeed, would be equivalent to a finding a solution for the low-energy limit of QCD and perhaps to confinement. In the absence of such solutions, one must rely on more elaborate effective Lagrangians but in doing so one faces problems: (i) the number of possible extensions, i.e. the number of possible terms at higher orders becomes increasingly large, (ii) the degree of the equation of motion becomes infinite and, (iii) adding terms of all orders introduces an arbitrarily large number of parameters and one loses any predictive power. Any one of these problems causes the general approach to become too complex and its treatment intractable.

There is however an alternative. One can impose some kind of symmetry or constraint that reduces this degree of arbitrariness and complexity but still retain some interesting new features. The idea is not new. Indeed, the second term in Skyrme Lagrangian is not the most general one but Skyrme justified this choice by arguing that this is the only combination which is of degree two, at most, in the derivative with respect to time. This term however stabilize the soliton and although it is of order four, it leads to a nonlinear differential equation of degree two for the static solution having a hedgehog form. Obviously, the same constraint cannot be implemented to orders larger then eight if one wants to preserve Lorentz covariance. Recently, we proposed a different approach to construct tractable allorders effective Lagrangian [3]: Using the hedgehog ansatz for static solution, we required that the degree of the differential equation remains two. This defines a class of all-orders Skyrmions with a number of interesting properties: (i) they are more conform to reality since they come from all-orders effective Lagrangians, (ii) the Lagrangians are chirally invariant by construction which will eventually allow more control over symmetry breaking terms since they can be added by hand afterwards, (iii) the physical predictions can be significantly improved [4], (iv) their topological and stability properties are similar and (v) they have been shown to have new interesting features such as a two-phase structure [5].

In this work, we identify two more class of tractable all-orders Skyrmions. This is done in section II, where we also find the explicit form of the Lagrangians from which they originate. The next section looks at toy models that can be solved analytically. Although their physical interpretation is not obvious some of them carry interesting properties like scale invariance of the soliton. Finally, the last section contains a brief discussion.

\section{ALL-ORDERS SKYRMIONS}




\section{A. A class of all-orders Skyrmions}

The Skyrme model is based on the chirally invariant Lagrangian

$$
\mathcal{L}_{\text {Skyrme }}=-\frac{f_{\pi}^{2}}{4} \operatorname{Tr} L_{\mu} L^{\mu}+\frac{\epsilon^{2}}{4} \operatorname{Tr}\left[L_{\mu}, L_{\nu}\right]^{2}
$$

where $L_{\mu}=U^{\dagger} \partial_{\mu} U$ is the left-handed current and $U$ is an $\mathrm{SU}(2)$ matrix related to the pion fields by the $U \equiv(\sigma+i \tau \cdot \pi) / f_{\pi}$. Two parameters are required, $f_{\pi}$, the pion decay constant $(93 \mathrm{MeV})$, and $\epsilon$ a dimensionless constant. The first and second term of this expression is the nonlinear $\sigma$-model Lagrangian and a stabilizing term added by Skyrme respectively.

The class of effective Lagrangians that we are interested in are constructed out of powers of the left-handed current $L_{\mu}$, more precisely, from powers of the commutators $f_{\mu \nu} \equiv\left[L_{\mu}, L_{\nu}\right]$. For example, the lowest order contributions may have the form

$$
\operatorname{Tr} f_{\mu \nu} f^{\mu \nu}, \quad \operatorname{Tr} f_{\mu}{ }^{\nu} f_{\nu}{ }^{\lambda} f_{\lambda}{ }^{\mu}, \quad \operatorname{Tr}\left(f_{\mu \nu} f^{\mu \nu}\right)^{2}, \quad \operatorname{Tr} f_{\mu}{ }^{\nu} f_{\nu}{ }^{\lambda} f_{\lambda}{ }^{\sigma} f_{\sigma}{ }^{\mu}, \quad \text { etc... }
$$

The choice of such combinations is motivated by the possibility that they could be induced by hidden gauge symmetry (HGS) terms [6, 3, 7]. They are also automatically chirally invariant. Chiral symmetry breaking must be added independently, usually by adding a pion mass term, which means that one has in principle more control on the symmetry breaking mechanism. The Skyrme term itself emerges from the gauge field kinetic term in the limit of large gauge vector mass in this formulation.

Using the hedgehog ansatz for the static solution, i.e. $U=\exp [i \tau \cdot \widehat{r} F(r)]$ where $F(r)$ is the chiral angle, a number of properties of the traces were found (see ref. 四):

(i) All traces are polynomials of the following combinations, $a \equiv r^{-2} \sin ^{2} F$ and $b \equiv F^{2}$. They can be written as

$$
\operatorname{Tr}\left(f_{\mu \nu}\right)^{n}=\sum_{m=0}^{M} \kappa_{n, m} a^{n-m}(b-a)^{m}
$$

where $\left(f_{\mu \nu}\right)^{n}$ represents a linear combination of any Lorentz invariant product of $n$ $f_{\mu \nu}$ 's and $M=\left[\frac{n}{2}\right]$ is the integer part of $\frac{n}{2}$.

(ii) The constants $\kappa_{n, m}$ are such that the ration of $\kappa_{n, 0}$ over $\kappa_{n, 1}$ is found to be $\frac{3}{n}$. For example,

$$
\operatorname{Tr} f_{\mu \nu} f^{\mu \nu}=16 a[a+2 b], \quad \operatorname{Tr} f_{\mu \nu} f^{\nu \lambda} f_{\lambda}^{\mu}=96 a^{2} b, \ldots
$$

where the first expression corresponds to the Skyrme term. Note that $\operatorname{Tr} L_{\mu} L^{\mu}$ in (1) also gives a similar result, i.e. $\operatorname{Tr} L_{\mu} L^{\mu}=2(2 a+b)$. Furthermore, one can construct a special class [3] of such combinations which is at most linear in $b$ (or of degree two in derivatives of $F$ ). These Lagrangians give a very simple form for the hedgehog solution

$$
\operatorname{Tr}\left(f_{\mu \nu}\right)^{n}=\kappa_{n, 0} a^{n-1}[3 a+n(b-a)]
$$

and lead to a chiral angle equation which is tractable since it is of degree two. It also turns out that for this class of Lagrangians $\kappa_{n, 0}=0$ for $n$ odd $\geq 5$. 
The energy of the static solution (or mass of the soliton) is then given by the general expression:

$$
\mathcal{M}_{S} \equiv-\int d^{3} r \mathcal{L}=4 \pi \int r^{2} d r \sum_{n=1}^{\infty} \sum_{m=0}^{M} \kappa_{n, m} a^{n-m}(b-a)^{m}
$$

The special class of combinations which is at most linear in $b$ gives an even simpler form since $\kappa_{n, m}=0$ for $m>1$ :

$$
\mathcal{M}_{S}=4 \pi \int r^{2} d r\left[3 \chi(a)+(b-a) \chi^{\prime}(a)\right]
$$

where $\chi(x)=\sum_{n=1}^{\infty} c_{n} x^{n}$ and $\chi^{\prime}(x)=d \chi(x) / d x$. Note that for $\mathcal{M}_{S}$ to be positive, it is sufficient (but necessary) to impose the conditions $c_{n} \geq 0$ and $(b-a) \geq 0$. Minimizing the static energy with respect to the chiral angle leads to differential equation:

$$
\chi^{\prime}(a)\left(F^{\prime \prime}+2 \frac{F^{\prime}}{r}-2 \frac{\sin F \cos F}{r^{2}}\right)+a \chi^{\prime \prime}(a)\left(F^{\prime 2} \cot F-2 \frac{F^{\prime}}{r}+\frac{\sin F \cos F}{r^{2}}\right)=0
$$

where again $a \equiv r^{-2} \sin ^{2} F$.

The existence of solutions requires that the series $\chi(a)$ converges for all values of $r$, leading to one can call summable Skyrmions. So it is closely related to the scaling properties of the Lagrangian. Order by order the terms scales according to

$$
\mathcal{M}_{S} \equiv-\int d^{3} r \mathcal{L}=\alpha_{2} R+\alpha_{4} R^{-1}+\alpha_{6} R^{-3}+\cdots+\alpha_{n} R^{3-n}+\cdots
$$

when $r$ is scaled according to $r \rightarrow r R^{-1}$ and $2 n$ is the number of derivatives. Obviously, the first term prevent the soliton from exploding. The exact behavior as $R \rightarrow 0$ and the existence of a global minimum (and of a Skyrmions) depend on the details of the series $\chi(x)$. The existence of Skyrmions has been demonstrated for a number of models for which it is also possible to compute baryon static properties [4, 5, 3] 8 using usual methods. In some cases, the solutions have shown an interesting two-phase structure. This last behavior is related to the convergence of $\chi(x)$.

The Lagrangians in (4) must be constructed by hand, order by order. But the contribution to the static energy obeys a recursion relation. If $\mathcal{M}_{n}=a^{n-1}[3 a+n(b-a)]$ is the static energy coming from order $2 n$, then the $n^{\text {th }}$ contribution could be obtain from

$$
\mathcal{M}_{n}=-\mathcal{M}_{n-2} \mathcal{M}_{2}+\mathcal{M}_{n-4} \mathcal{M}_{4}-\frac{1}{3} \mathcal{M}_{n-6} \mathcal{M}_{6}
$$

for even $n \geq 8$.

Finally, we mention that similar results hold for constructions of the form $\operatorname{Tr}\left(L_{\mu}\right)^{2 n}$ but in this case $M=n$ in the expression in (何) and in general, $\kappa_{n, 0} \neq 0$ for $n$ odd $\geq 5$. A corresponding recursion relation, which applies in this case to all $n>4$, can also be written.

\section{B. New classes}

In light of those results we propose two other classes of all-orders Skyrmions. First, let us rewrite equation (3), 


$$
\operatorname{Tr}\left(f_{\mu \nu}\right)^{n}=\kappa_{n, 0}\left[a^{n}+\left(2 a^{n}+n a^{n-1}(b-a)+\cdots+\frac{\kappa_{n, M}}{\kappa_{n, 0}} a^{M}(b-a)^{M}\right)\right] .
$$

where $M=\left[\frac{n}{2}\right]$. As mentioned above, it is possible to combine several traces of order $n$ and eliminate higher orders in $b$ and obtain a class of summable Skyrmions. But the last expression also suggest another interesting case where the traces combine in a way that the term in parenthesis corresponds to the binomial expansion. It simplifies as follows:

$$
\left.\operatorname{Tr}\left(f_{\mu \nu}\right)^{n}=\kappa_{n, 0}\left[a^{n}+2 a^{M}(a+(b-a))^{M}\right)\right]=\kappa_{n, 0}\left[a^{2 M}+2(a b)^{M}\right]
$$

for $n$ even and $M=\frac{n}{2}$.

This second class of all-orders Skyrmions is then characterized by the static energy

$$
\mathcal{M}_{S}=4 \pi \int r^{2} d r[\chi(a)+2 \chi(\sqrt{a b})]
$$

where $\chi(x)=\sum_{n=1}^{\infty} c_{n} x^{n}$ with $c_{n}=0$ for $n$ odd $>3$. Here for $\mathcal{M}_{S}$ to be positive definite, it is sufficient that the model obeys $c_{n} \geq 0$. The chiral angle is then described by:

$$
2 \chi^{\prime}(a) \cos F-\chi^{\prime}(\sqrt{a b})-\chi^{\prime \prime}(\sqrt{a b})\left(F^{\prime 2} \cos F+F^{\prime \prime} \sin F-F^{\prime} \frac{\sin F}{r}\right)=0
$$

where again $a \equiv r^{-2} \sin ^{2} F$ and $b \equiv F^{\prime 2}$. This second class of Lagrangians is generated order by order by the systematic procedure (see ref. [4] for details):

$$
\operatorname{Tr}\left(f_{\mu \nu}\right)^{n}=\operatorname{Tr}\left[\left\{f_{\mu}{ }^{\nu}, f_{\lambda}{ }^{\rho}\right\}\left\{f_{\nu}{ }^{\lambda}, f_{\sigma}{ }^{\omega}\right\}\left\{f_{\rho}{ }^{\sigma}, f_{\xi}{ }^{\eta}\right\}\left\{f_{\omega}{ }^{\xi}, f_{\eta}{ }^{\beta}\right\} \cdots\right] .
$$

The third class of all-orders Skyrmions comes on the other hand from Lagrangians build in terms of even powers of $L_{\mu}$ 's instead of powers of $f_{\mu \nu}$ 's. In this case, the traces contains terms up to $O\left(b^{n}\right)$ and can be written

$$
\operatorname{Tr}\left(L_{\mu}\right)^{2 n}=\kappa_{n, 0}\left[2 a^{n}+\left(a^{n}+n a^{n-1}(b-a)+\cdots+\frac{\kappa_{n, n}}{\kappa_{n, 0}} a^{n}(b-a)^{n}\right)\right] .
$$

Following equation (10), we choose the combination for which the term in parenthesis correspond to the binomial expansion of $(a+(b-a))^{n}$ and find

$$
\operatorname{Tr}\left(L_{\mu}\right)^{2 n}=\kappa_{n, 0}\left(2 a^{n}+b^{n}\right) .
$$

The explicit structure of traces leading to the above result is the a simple product of the anticommutator defined by $d_{\mu \nu} \equiv\left\{L_{\mu}, L_{\nu}\right\}$, i.e.

$$
\operatorname{Tr} d_{\mu}^{\nu} d_{\nu}^{\lambda} d_{\lambda}^{\sigma} d_{\sigma}^{\alpha} \cdots d_{\rho}^{\mu}=\kappa_{n, 0}\left(2 a^{n}+b^{n}\right) .
$$

The static energy takes a simple form

$$
\mathcal{M}_{S}=4 \pi \int r^{2} d r[2 \chi(a)+\chi(b)]
$$

where $\chi(x)=\sum_{n=1}^{\infty} c_{n} x^{n}$. For $\mathcal{M}_{S}$ to be positive definite, it is sufficient that $c_{n} \geq 0$. The chiral angle must now obey: 


$$
2 \chi^{\prime}(a) \sin F \cos F-\chi^{\prime}(b)\left(2 r F^{\prime}+r^{2} F^{\prime \prime}\right)-2 \chi^{\prime \prime}(b) r^{2} F^{\prime 2} F^{\prime \prime}=0
$$

This last expression takes a very simple form if we define $\zeta(x) \equiv \chi\left(x^{2}\right)=\sum_{n=1}^{\infty} c_{n} x^{2 n}$ since the arguments of $\chi$ are squared objects (either $a$ or $b$ ). The equation becomes

$$
2 \zeta^{\prime}\left(\frac{\sin F}{r}\right) r \cos F-2 \zeta^{\prime}\left(F^{\prime}\right)-\zeta^{\prime \prime}\left(F^{\prime}\right) r^{2} F^{\prime \prime}=0 .
$$

The results in (7), (12) and (18) can be considered as special cases. In general, the equation for the chiral angle should depends on a more complicated functional , $\chi(a, b)$, to take into account the general expression in (9).

\section{TOY MODELS WITH ANALYTICAL SOLUTIONS}

Instead of trying to find solutions for each class of model, we adopt a different point of view here. We look at special solutions and try to find what model can accommodate it at the expense of physical meaning. The interest of the procedure will become more clear below. For this purpose, we will assume that the function $\chi(x)$ is no longer constrained to have a power series representation so are not directly related to the aforementioned classes of Lagrangians. Looking at the chiral angle equations described above its becomes apparent that a solution which obeys

$$
F^{\prime}=-\alpha \frac{\sin F}{r},
$$

simplifies the chiral angle equation for all class of models. Since we are interested in the $\mathrm{N}=1$ soliton with $F(0)=\pi$ and $F(\infty)=0$, the constant $\alpha$ must be positive. The solution for $F$ is simply:

$$
F(r)=2 \arctan \left(a_{1} r^{-\alpha}\right)
$$

Since the static energy must be finite, $F$ is required to decrease fast enough as $r \rightarrow \infty$. Clearly this imposes an extra condition on the range of possible values of $\alpha$.

Imposing this solution for the chiral angle equations in (7), (12) and (18) translate into differential equations for $\chi(x)$. In the first case, equation (7) becomes

$$
\left(\alpha^{2}-1\right) x \chi^{\prime \prime}(x)=-\left(3-2 \alpha^{2}\right) \chi^{\prime}(x) .
$$

which can be solved for $\chi(x)$ :

$$
\chi(x)=C_{1}+\frac{C_{2}}{\beta} x^{\beta}
$$

with $\beta=\frac{3 \alpha^{2}-4}{\alpha^{2}-1}$. Physically plausible solution requires that $C_{1}=0$ because of the finiteness of the static energy. The remaining model corresponds to a Lagrangian with $2 \beta$ in derivatives of the pion field. In order to make any sense $2 \beta$ must be an integer, otherwise one is forced to consider fractional power of a derivative. From scaling argument, it is easy to see that for $\beta<\frac{3}{2}$ the "size" of the solution collapse to zero, for $\beta>\frac{3}{2}$, energy favors infinite size configuration and the solution eventually disperse. Therefore none of those solution are stable solitons. However for $\beta=\frac{3}{2}$, the solution is scale invariant. Among the values that $\beta$ may take, three of them seem of particular interest: 
(i) $\alpha=\sqrt{\frac{3}{2}}$ : For this value of $\alpha, \beta=1$ and the model correspond to the nonlinear $\sigma$-model. The solution is therefore not stable under scale transformations but at least one can attach a physical meaning to the model.

(ii) $\alpha=1$ : This solution correspond to the limit of $\beta \rightarrow \infty$, i.e. the only contribution comes from a term with an infinite number of derivatives. The solution is not a stable Skyrmion.

(iii) $\alpha=\sqrt{\frac{5}{3}}$ : Since $\beta=\frac{3}{2}$, the static energy given by this model

$$
\mathcal{M}_{S}=4 \pi C_{2} \int r^{2} d r\left(\frac{\sin ^{2} F}{r^{2}}\right)^{\frac{1}{2}}\left[\frac{\sin ^{2} F}{r^{2}}+F^{\prime 2}\right]
$$

is invariant under scaling. Using the solution, we can rewrite the static energy

$$
\mathcal{M}_{S}=-4 \pi C_{2} \cdot \frac{8}{\sqrt{15}} \cdot \int_{0}^{\infty} d r F^{\prime} \sin ^{2} F=\frac{16 \pi^{2} C_{2}}{\sqrt{15}} \cdot N
$$

and we see that result is proportional to the topological number, $N=1$ here. The connection between this model and any physically relevant Lagrangian is not obvious, but the mere fact that the static energy is proportional to the topological (or baryon) number is in itself rather interesting.

The second class of summable Skyrmions, described by equation (12), requires some care. In order to simplify the differential equation, we will assume that $\chi$ takes a form similar to (21). For this choice to be consistent one must then look at the negative root of $\sqrt{a b}$, i.e.

$$
\sqrt{a b}=-\alpha \frac{\sin ^{2} F}{r^{2}}
$$

in which case $\beta=\frac{1}{2}$, and $\alpha$ is found to be 4 for the $N=1$ soliton solution. If we were to associate this model to a Lagrangian we would have to construct one with a single, i.e. $2 \beta=1$, derivative of the field. In any case, there is no stable Skyrmion here but the energy of this unstable solution takes the simple form

$$
\mathcal{M}_{S}=24 \pi C_{2} \int d r r \sin F=\frac{48 \pi}{\sqrt{a_{1}}} C_{2} \cdot \frac{\pi}{4 \sqrt{2}}
$$

Finally, the equation for the third class of summable Skyrmions is given by (17) and for $\alpha=1$, it becomes

$$
2 x \chi^{\prime \prime}(x)=\chi^{\prime}(x) .
$$

Solving for $\chi(x)$, we get the form (21) with $\beta=\frac{3}{2}$ (and $C_{1}=0$ ). The function $\chi(x)$ is identical to the one found in the first class with the choice $\alpha=\sqrt{\frac{5}{3}}$. This means that the static energy is again invariant under scaling. Although the static energy can be recast in a form similar to (23), i.e. proportional to the topological number, the chiral angle is clearly not the same:

$$
F(r)=2 \arctan \left(a_{1} r^{-1}\right)
$$

and the two models are completely different. 


\section{DISCUSSION AND CONCLUSIONS}

We have introduce two new classes of summable Skyrmions and have written the Lagrangians which induce them. The basic elements for solving the chiral angle equation are in place and once the solution is found (by numerical analysis usually) the physical quantities can computed in a straightforward procedure similar to the first class of models. There remains however a noumber of open question. One must verify that solution can be reach for any plausible model and provide examples where these new summable Skyrmions indeed exists. The second point that would be interesting to analyse is the possibility of a phase structure. In the first class of model, the phase structure is related to the convergence of the series for $\chi(a)$. In the second and third classes $\chi(\sqrt{a b})$ and $\chi(a b)$, respectively, are also required to converge otherwise the solution has infinite energy. The extra requirement may have some intriguing effects on the solutions. It may well be that it will introduce a solution which experience three phases, one dominated by small $a$ and $b$ a second one regulated by the convergence of $\chi(a)$ and a new phase regulated by the convergence of $\chi(\sqrt{a b})$ or $\chi(a b)$. One can also imagine another alternative: a bifurcation between two solutions each having a two-phase structure, the first solution would correspond to $\chi(a)$ and the second, to $\chi(\sqrt{a b})$ or $\chi(a b)$. In principle the two solutions could even be degenerate. Since there is still a large degree of freedom in the construction of physically viable models none of these effects are excluded a priori. Finally, the question of quantum stability must also be addressed for these new classes of models.

Finally, we have also found analytical solution to toy models in this work. They are motivated by the relations that were obtained for the class of summable Skyrmions but no longer carries any relations with the power series representation or with the Lagrangian constructed in section II, and so, their physical interpretation is unclear. However the main conclusion that comes out of this exercise is that the stable annalytical solutions (solitons) that were found are scale-invariant solutions and their static energy proportional to the topological number which is a feature also observed in the sine-Gordon model in $(1+1)$ space-time dimensions.

\section{ACKNOWLEDGEMENT}

The author would like to thank T.N. Truong for the hospitality of the Centre de Physique Théorique where part of this work was done. This research was supported by the Natural

Science and Engineering Research Council of Canada and by the Fonds pour la Formation de Chercheurs et l'Aide à la Recherche du Québec. 


\section{REFERENCES}

$\dagger$ On sabbatical leave from: Département de Physique, Université Laval, Québec, Canada, G1K 7P4.

[1] T.H.R. Skyrme, Proc. R. Soc. London. A2603, 127 (1961).

[2] G. 't Hooft, Nucl. Phys. B72, 461 (1974); E. Witten, Nucl. Phys. B160, 57 (1979).

[3] L. Marleau, Phys. Lett. B235, 141 (1990).

[4] S. Dubé and L. Marleau, Phys. Rev. D41, 1606 (1990); L. Marleau, Phys. Rev. D43, 885 (1991).L. Marleau, Phys. Rev. D45, 1776 (1992).

[5] K. Gustafsson and D.O. Riska, Two-phase structure of infinite order skyrmion, University of Helsinki preprint HU-TFT-93-11, January 1993.

[6] M. Bando, T. Kugo, S. Uehara, K. Yamawaki and T. Yanagida, Phys. Rev. Lett. 541215 (1985); M. Bando, T. Kugo and K. Yamawaki, Phys. Rep. 164217 (1988).

[7] L. Marleau and H. Omari, Phys. Rev. D48, (1993).

[8] A.D. Jackson, C. Weiss and A. Wirzba, Nucl. Phys. A529, 741 (1991). 